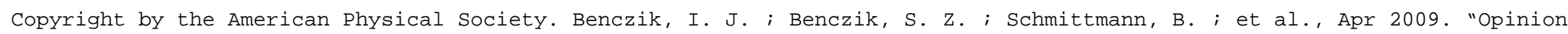
dynamics on an adaptive random network," PHYSICAL REVIEW E 79(4): 046104. DOI: 10.1103/PhysRevE.79.046104.

\title{
Opinion dynamics on an adaptive random network
}

\author{
I. J. Benczik, S. Z. Benczik, B. Schmittmann, and R. K. P. Zia \\ Department of Physics, Virginia Tech, Blacksburg, Virginia 24061-0435, USA
}

(Received 30 December 2008; published 6 April 2009)

\begin{abstract}
We revisit the classical model for voter dynamics in a two-party system with two basic modifications. In contrast to the original voter model studied in regular lattices, we implement the opinion formation process in a random network of agents in which interactions are no longer restricted by geographical distance. In addition, we incorporate the rapidly changing nature of the interpersonal relations in the model. At each time step, agents can update their relationships. This update is determined by their own opinion, and by their preference to make connections with individuals sharing the same opinion, or rather with opponents. In this way, the network is built in an adaptive manner, in the sense that its structure is correlated and evolves with the dynamics of the agents. The simplicity of the model allows us to examine several issues analytically. We establish criteria to determine whether consensus or polarization will be the outcome of the dynamics and on what time scales these states will be reached. In finite systems consensus is typical, while in infinite systems a disordered metastable state can emerge and persist for infinitely long time before consensus is reached.
\end{abstract}

DOI: 10.1103/PhysRevE.79.046104

PACS number(s): 89.75.Hc, 02.50.Le, 05.70.Ln, 89.65.Ef

\section{INTRODUCTION}

Models from statistical physics have been invoked successfully to model social phenomena, such as opinion formation, voting preferences, and the spreading of information or disease [1]. All of these phenomena involve a large number of interacting degrees of freedom ("agents") which evolve dynamically in time, subject to external driving forces and some form of noise. The emergence of final states, subject to different initial states and driving forces, can often be understood using concepts from nonlinear dynamics, critical phenomena, and phase transitions. Methods from nonequilibrium statistical dynamics have been especially useful since the dynamics underlying these phenomena typically violate detailed balance (or equivalently, microscopic reversibility). As a consequence, the long-time behavior of these models falls outside the usual equilibrium framework.

In the following, we investigate a simple model for opinion formation in a two-party system, based on a variation of the voter model. The voter model [2] has attracted much attention due to several features. First, from a theoretical perspective it is one of the very few stochastic many-body systems that are solvable on lattices of any dimension. Thus, it serves as a benchmark for simulations or perturbative solutions of more complex models. Second, it has broad interdisciplinary applications. In chemistry, it has been invoked to describe the kinetics of catalytic reactions $[3,4]$ or for studying coarsening phenomena $[5,6]$. In population dynamics and evolutionary biology, it describes the competition of species $[7,8]$. Beyond the framework of the sciences, voter and voterlike models have found many applications to phenomena which rely on human behavior, e.g., the emergence of collective organization in sociocultural situations [9-26].

In sociophysics, the voter model combined with wellknown tools from statistical physics, such as various meanfield-like approaches and exact methods [9,10,14,19,24,25] or numerical simulations $[12,15,17,20,26]$, was used extensively to describe the spreading of culture, religion, languages, or political opinions in social systems. Even though the model neglects certain social factors, its appealing mathematical simplicity allows analytical investigations and can provide general predictions about such complex systems. More elaborate variants of the voter model, such as the Axelrod $[13,16,28]$ or Sznajd $[12,19]$ model, were also implemented to study particular types of opinion dynamics.

In physical situations, voter models and their variants have mainly been studied on homogeneous and translationally invariant spatial structures, i.e., regular lattices. In social contexts, studies on regular lattices [6,13-17] offer, at best, a rough approximation of geographic vicinity which generally leads to a spatial segregation of opinions. With recent advances in transportation and information technologies, however, social interactions are no longer limited by geographical distance so that the interaction patterns between individuals find a better characterization as complex networks. Indeed, in some recent attempts to formulate voter models on graphs $[18,19]$, random [20], scale free [21], or small world networks [22,23], some aspects of the complex connections between the members of a social system were taken into account.

Another issue that has not yet been addressed in depth is the variability of the connections-or relationshipsbetween individuals in time. The increased mobility of people and the plethora of new communication tools make it easy for agents to break or replace old connections and to generate new ones. Moreover, the actual opinions of two people will determine whether these two individuals will, or will not, establish a relationship so that the opinion dynamics takes place on an adaptively changing network. More precisely, the full dynamics of such a social network consists of (i) the individual's opinion formation process (taking place on the nodes) and (ii) the evolution of the underlying topological structure (links). The coupling between these two processes reflects how the connections between people influence their opinions and how their opinions determine, in turn, their new connections. This possible coevolution of nodes and links-i.e., of the full network structure-has been studied only in a few works [24-27,29-31]. 
Our aim is to propose a simple model in which voters have a choice between two political parties and the dynamics of opinion formation takes place on an adaptive random network characterized by (i) and (ii). In Sec. II, first we present a simple model for opinion dynamics on an adaptive network and discuss possible interpretations and the relevance of this particular model. In Sec. III we write a master equation for the dynamics of the system. The exact calculation of the transition rates appearing in the master equation is presented in the Appendix. In Sec. IV we solve this master equation exactly in the thermodynamic limit and verify the results by numerical simulations. Depending on the initial conditions and the parameters of the model, four final states are found: a disordered state, a completely frozen and (partially) polarized situation, or complete consensus aligned with the opinion of either one of the two parties. These last two states are absorbing-once the system reaches either one of them, it can no longer access any other configurations. We should emphasize that these states, and their names, refer to the individuals' opinions, since the links continue to be updated according to our dynamics. In Sec. $\mathrm{V}$ we discuss finite-size effects. For finite systems, only the absorbing states are truly stable while the other two states are metastable with finite (but very large) lifetimes. In Sec. VI, we generalize the model to more complex situations. We conclude with a summary and some open questions. A short summary of some of these results was already published in [32].

\section{MODEL}

We consider a network (graph) with $N$ nodes. Each node $j$ ("individual") carries a spin $\sigma_{j}$ ("opinion") which can take two different values $\sigma_{j}= \pm 1$, modeling the choice of two parties. In order to reflect the time-dependent, adaptive nature of linkages between individuals, we implement a dynamics in which links and nodes are coupled. At each time step, we choose a spin at random and update its links to all other nodes as follows. If the other node carries the same spin value (i.e., "shares the same opinion"), the link is established with probability $p$; with probability $1-p$, no link will be present between the two spins. Similarly, if the other node carries the opposite spin, a link is formed with probability $q$. In other words, pairs of nodes carrying equal (opposite) spins are connected with probability $p(q)$.

Immediately after the links are updated, the chosen spin is updated according to a simple majority rule, independent of its initial state. For example, if it is connected to more positive than negative spins, its state will be positive in the next time step regardless of its original state. In the case of a tie, the spin remains unchanged.

Since spins and links coevolve-i.e., the presence or absence of a link depends on the state of the spins on the link and the state of the spins depends on the presence or absence of links-we call the network adaptive. As an interpretation, we propose that this model mimics a two-party electoral system. During a campaign, the supporters of one party (characterized by $\sigma_{j}=+1$ ) are keen to interact with supporters of the other party (with $\sigma_{j}=-1$ ) to try to convince them and change their opinion. This situation can arguably be de- scribed by this model with $p<q$, when each agent has more interactions with opponents than with agents sharing the same opinion (according to the motto that "convinced people do not need to be convinced again"). On the other hand, when $p>q$, the supporters of any party tend to interact more with individuals sharing the same opinion (according to the motto "united we are stronger"). The latter behavior might be a simplified description of the process of political polarization, when many members of a given party agree with the official position of the party, as often occurs in post-election periods.

Our model is aimed to describe a free public debate in the sense that it does not consider the effects of central institutions or the mass media; neither lobbying nor organized strategies are taken into account. As a result, the model may also be appropriate to describe groups defined by criteria such as education, religion, or ethnicity rather than political opinion. Cultural assimilation, the spreading of a language or of a religion, rumor or fear propagation, and social reforms are examples of phenomena which can be modeled in this (simplified) fashion.

\section{MASTER EQUATION}

To describe the dynamics of the system in complete detail would require writing a master equation for the $N(N+1) / 2$ variables which fully characterize the state of the nodes and links. While this task is feasible, the resultant would be quite complicated. Instead, we note that the presence or absence of links between a given spin and the remainder of the system is controlled by simple binomial distributions. The detailed properties of these distributions, to be discussed below, determine whether this spin will be flipped in the next time step. Since spins flip one at a time, the dynamics of the nodes is simply a birth-death process for, e.g., the total number of positive spins, $M$. It can be characterized in terms of $P(M, t)$, the probability of finding the network with $M$ positive spins at time $t$. The average fraction of positive nodes is

$$
\rho(t) \equiv \sum_{M=0}^{N} \frac{M}{N} P(M, t)
$$

and can be regarded as the "popularity" of positive opinions at time $t$. This popularity takes values in the interval $[0,1]$, where $\rho=0.5$ characterizes the completely disordered state, while the extremes $(\rho=0,1)$ correspond to a complete ordering of the system, a state we will call "consensus." Contrary to the voter model defined on a regular lattice, the global magnetization (given by $m=2 \rho-1$ ) is not conserved here. Note that the dynamics is still $\mathbb{Z}_{2}$ symmetric, i.e., invariant under the global inversion $\sigma_{i} \rightarrow-\sigma_{i}$ (or simply $M / N \rightarrow 1-M / N)$.

The time evolution of $\rho$ is fully determined by the dynamics of $P(M, t)$, for which we derive a master equation. Since the spins flip one at a time and the state with $M$ positive spins can be reached by a single flip event only from the states with $M-1$ or $M+1$ positive spins, the time evolution of $P(M, t)$ is a simple birth-death process 


$$
\begin{aligned}
\partial_{t} P(M, t)= & b_{M-1} P(M-1, t)+d_{M+1} P(M+1, t) \\
& -\left[b_{M}+d_{M}\right] P(M, t),
\end{aligned}
$$

where $b_{M}$ denotes the birth rate of a positive spin (i.e., the rate for flipping a negative spin to positive) and $d_{M}$ its death rate (flipping from positive to negative). Both depend on $M$, the current number of positive spins in the system. As an example, we provide the details for evaluating $d_{M}$. First, it comes with a factor of $M / N$, reflecting the probability of finding a positive spin among the $N$ spins. Next, we ask for the probability that this spin is connected to exactly $k$ of the other $M-1$ positive spins and $k^{\prime}$ (of the $N-M$ ) negative ones. Since these connections are established randomly, with probabilities $p$ and $q$, respectively, the probability of such a set of connections is just a product of two binomial distributions, $B_{M-1, p}(k) B_{N-M, q}\left(k^{\prime}\right)$, where we have defined

$$
B_{L, p}(\ell) \equiv\left(\begin{array}{c}
L \\
\ell
\end{array}\right) p^{\ell}(1-p)^{L-\ell} .
$$

Since the selected positive spin will flip provided $k<k^{\prime}, d_{M}$ is just the sum over all possible pairs $\left(k, k^{\prime}\right)$ with the constraint $k<k^{\prime}$,

$$
d_{M}=\frac{M}{N} \sum_{k=0}^{M-1} \sum_{k^{\prime}=0}^{N-M} B_{M-1, p}(k) B_{N-M, q}\left(k^{\prime}\right) \Theta\left(k^{\prime}-k\right),
$$

where

$$
\Theta(\ell) \equiv\left\{\begin{array}{rr}
1 & \text { if } \ell>0 \\
0 & \text { otherwise } .
\end{array}\right.
$$

Similar considerations lead to the birth rate

$$
b_{M}=\frac{N-M}{N} \sum_{\ell=0}^{N-M-1} \sum_{\ell^{\prime}=0}^{M} B_{N-M-1, p}(\ell) B_{M, q}\left(\ell^{\prime}\right) \Theta\left(\ell^{\prime}-\ell\right) \text {. }
$$

For the special case $q=1-p$, the $\Theta$ functions can be eliminated from $b_{M}$ and $d_{M}$ (due to the properties of binomial distributions; details can be found in the Appendix) to yield

$$
\begin{gathered}
d_{M}=\frac{M}{N} \sum_{n=0}^{N-M-1} B_{N-1, p}(n), \\
b_{M}=\frac{N-M}{N} \sum_{m=0}^{M-1} B_{N-1, p}(m) .
\end{gathered}
$$

These rates depend in a nontrivial way on the current number of positive and negative spins in the system and thus embody the adaptivity of the network. They also depend on the probabilities $p$ and $q$, and on the size of the system. The latter dependence is illustrated in Fig. 1 where the upper (lower) panels show the rates for $N=1000(N=50)$.

In the remainder of this paper, we present solutions of the master equation, given an initial distribution

$$
P(M, 0)=\delta\left(M, M_{0}\right),
$$

and so

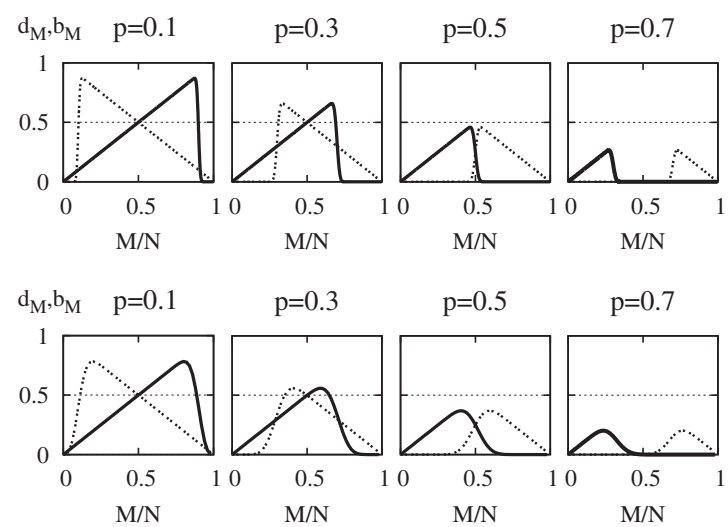

FIG. 1. The death $\left(d_{M}\right)$ and birth $\left(b_{M}\right)$ rates (solid and dashed lines, respectively) given by Eqs. (7) and (8) for different probabilities $p$ and $q=1-p$. Upper panels $N=1000$, lower panels $N=50$. For fixed $p$, there are three different regimes of behavior bounded by Eq. (11).

$$
\rho(0)=M_{0} / N \equiv \rho_{0} .
$$

First, we will focus on the special case $q=1-p$, with transition rates given by Eqs. (7) and (8). In Sec. VII we address briefly the general case when the rates are given by Eqs. (4) and (6).

\section{SYSTEMS WITH LARGE $N$}

In order to appreciate easily the different types of behavior and the parameter regimes where they occur, let us first consider the thermodynamic limit, $N \rightarrow \infty$. The solutions found here will guide our analysis of finite-size effects in Sec. V. For large $N$, the binomial distribution approaches a normal distribution. Thus the sum in Eq. (8) is given by the cumulative distribution function of the normal distribution, i.e., the Gaussian error function (shown as a solid line in Fig. 2(b)), and the birth rate $b_{M}$ is given by

$$
b_{M} \simeq \frac{N-M}{N} \Theta(M-N p)
$$
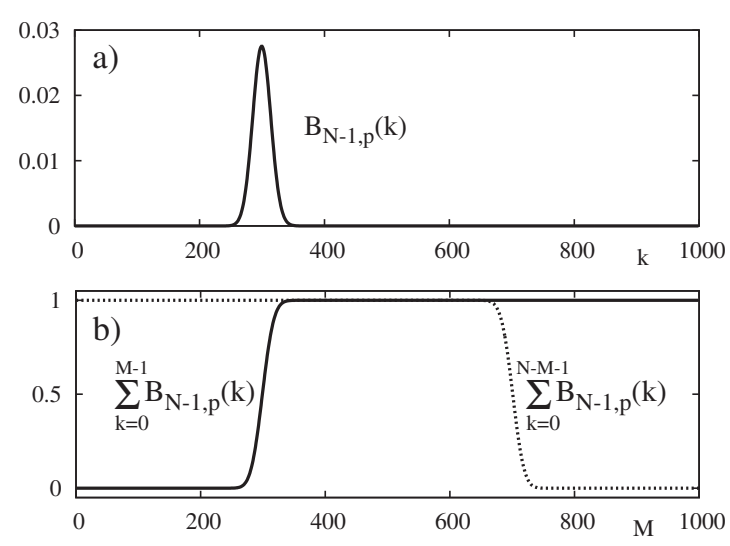

FIG. 2. (a) The binomial distribution $B_{N-1, p}(k)$ for $N=1000$ and $p=0.3$ approaches a Gaussian centered at $N p=300$. (b) The cumulative distribution functions appearing in Eqs. (8) and (7) (solid and dotted lines, respectively). 
apart from a region of width $\sqrt{N p(1-p)}$ around $N p$. The death rate $d_{M}$ is described by the complementary error function [the dotted line in Fig. 2(b)] and given by

$$
d_{M} \simeq \frac{M}{N} \Theta[N(1-p)-M]
$$

apart from a region of the same width as above around $N(1-p)$.

These simplified forms of the transition rates (see the upper panels in Fig. 1 for large $N$ ) allow us to determine easily the late-time properties of the model. For fixed $p$ there are three different regimes, depending on whether $b_{M}>d_{M}$, $b_{M}<b_{M}$, or $b_{M} \simeq d_{M} \simeq 0$. Starting with a value, $\rho_{0}$, for the initial density of positive spins, the panels in Fig. 1 allow us to determine whether this value will grow $\left(b_{M}>d_{M}\right)$, decrease $\left(b_{M}<d_{M}\right)$, or stay approximately constant $\left(b_{M} \simeq d_{M} \simeq 0\right)$. The boundaries between these regimes can be obtained from Eqs. (9) and (10) as

$$
\rho_{0}=p, \quad \text { and } \quad \rho_{0}=1-p .
$$

For $p<0.5$ and $\rho_{0}<p$, we find that $M / N$ stays below $p$ at later time also. Indeed, in the approximations (9) and (10), we have a pure death process

$$
\partial_{t} P(M, t)=(M+1) P(M+1, t)-M P(M, t),
$$

which leads to the extinction of the positive population. The solution of this master equation shows that the steady state, $\rho_{\infty} \equiv \lim _{t \rightarrow \infty} \rho(t)=0$, is reached exponentially as

$$
\rho(t) \sim \rho_{0} \exp (-t) .
$$

Similarly, if $p<0.5$ and $\rho_{0}>1-p$, we have a pure birth process and the system relaxes exponentially to the state $\rho_{\infty}=1$ on the same characteristic time scale (due to the $\mathbb{Z}_{2}$ symmetry).

In the intermediate region $\rho_{0} \in[p, 1-p]$, the dynamics is described by

$$
\begin{aligned}
\partial_{t} P(M, t)= & (M+1) P(M+1, t) \\
& +(N-M+1) P(M-1, t)-N P(M, t),
\end{aligned}
$$

and the system reaches a disordered phase: $\rho_{\infty}=0.5$. Again, the relaxation is exponential, with a characteristic time scale $1 / 2$,

$$
\rho(t)-\rho_{\infty}=\left(\rho_{0}-\rho_{\infty}\right) \exp (-2 t) .
$$

For finite systems, only the absorbing states can be true steady states of the system, and so this state should be metastable. However, its lifetime is enormous, being $O\left(e^{N}\right)$, and will be studied further in Sec. V.

For $p>0.5$, the pure death and pure birth regimes are the same as for $p<0.5$. A small minority $\left(\rho_{0}<1-p\right)$ will become extinct; a large majority $\left(\rho_{0}>p\right)$ will win. However, a new feature appears in the interval $\rho_{0} \in[1-p, p]$, where the system seems to acquire infinite memory. Both the death and birth rates vanish in this region, according to the approximations (9) and (10), so that the master equation reduces to
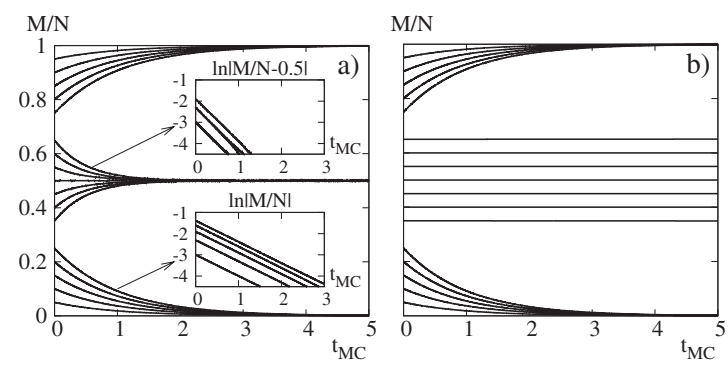

FIG. 3. Time evolution of the fraction $M / N$ of positive spins for a network of $N=1000$ nodes, for (a) $p=0.3$ and (b) $p=0.7$. The values are averaged over 1000 runs. Time is measured in Monte Carlo steps, $t_{\mathrm{MC}}=t_{\mathrm{spin}-\text { flip }} / N$. Insets show the exponential relaxation to the final states.

$$
\partial_{t} P(M, t)=0
$$

whence the fraction of positive spins remains frozen at its initial value

$$
\rho(t)=\rho_{0} .
$$

This behavior is understandable. If an individual "talks" only very rarely to those with the opposite opinion, essentially no changes can take place and the society remains "static." As in the case of $p<0.5$, this is also a metastable state for finite systems, with a lifetime of $O\left(e^{N}\right)$.

Our analytic findings are tested by direct simulations [33] on a network with $N=1000$ nodes. The relaxation into the late-time behaviors is clearly displayed in Fig. 3(a) for $p<0.5$ and Fig. 3(b) for $p>0.5$. Insets show the relaxations of type (13) to the absorbing state and of type (15) to the disordered state. Note that in our simulations Monte Carlo time $t_{\mathrm{MC}}$ is incremented only after every node has had a chance to flip, so that $t_{\mathrm{MC}}=t / N$.

To illustrate our findings further, Fig. 4 shows the outcome of the voter dynamics for two fixed initial fractions of the positive population: one starting from a minority $\rho_{0}<0.5$ and one starting from a majority $\rho_{0}>0.5$. For small $p$, the system reaches a disordered state, independent of $\rho_{0}$. The "open mindedness" of the population (reflected by a large probability $1-p$ to communicate with the opposite party) leads to an equal distribution of opinions. In contrast, an "inflexible attitude" (characterized by a large $p$ of linking up with similar opinions) leads to a fixed, unchanging distribution of opinions. For intermediate values of $p$, the system reaches a completely ordered state: all voters reach the same opinion, namely, that of the initial majority.

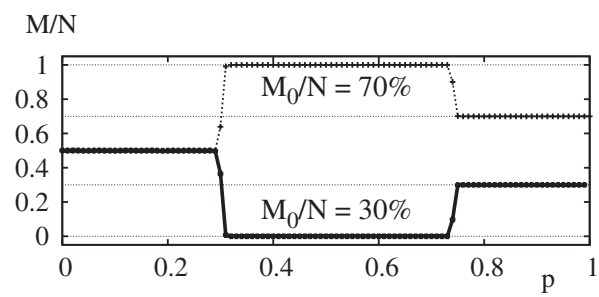

FIG. 4. Outcome of the voter dynamics as function of $p$ for two values of $M_{0} / N$. Solid (dotted) line shows $M_{0} / N=0.3(0.7)$. 


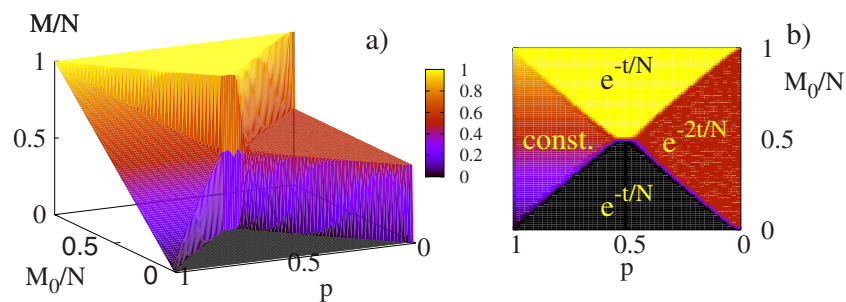

FIG. 5. (Color online) The fraction of positive spins $M / N$ after $t_{\mathrm{MC}}=10$, as function of $p$ and $M_{0} / N$ for a network of $N=1000$ nodes in a (a) three-dimensional plot and a (b) two-dimensional projection displaying clearly the phase transitions.

Summarizing our findings so far, we conclude that there are four distinct regimes, depending on the relative magnitudes of $\rho_{0}$ and $p$. In a $\left(p, \rho_{0}\right)$ phase diagram, these different regimes are bounded by Eq. (11). The numerical results for the possible outcomes of the voter dynamics on the adaptive network, for all parameters $p$ and initial fractions $\rho_{0}$ of the positive population, are shown in Fig. 5. The four different regimes can be characterized as follows: a perfect balance of opinions, a completely static situation, or perfect consensus (all positive or all negative). In Sec. V, we will discuss how these findings are modified in finite systems. Clearly, the two completely ordered states $(\rho=0,1)$ are absorbing states. Thus, they will eventually be reached from the other two (metastable) states. Two interesting questions remain. First, in which of the two absorbing states will each metastable state arrive, and second, how do the relaxation times depend on system size?

\section{FINITE-SIZE EFFECTS}

Generally, there are two time scales in these systems. On short time scales, the outcome of the voter dynamics follows our discussion above and can be illustrated by Fig. 5. In this section, this will be termed the short time scale behavior of the model.

On long time scales, however, the only possible steady states of the systems are the completely ordered absorbing states. To appreciate which of the two absorbing states is reached by the system after long times, we directly iterate the original master equation, Eq. (2), for the specific case of $N=100$. Aiming to obtain the final outcome of the voter dynamics, we evaluate the probabilities $P(M, \infty)$ for all $M \in[0,100]$. We choose parameters $p=0.3$ and $p=0.7$, as well as starting with all initial conditions $M_{0} \in[0,100]$. Not surprisingly, apart from $M=0$ and 100 , all probabilities $P(M, \infty)$ vanish, so that the final state can be characterized by just one probability, say, $P(N, \infty)$. Clearly, $P(0, \infty)=1-P(N, \infty)$. The results are displayed in Fig. 6, where $P(N, \infty)$ is shown as a function of the initial $\rho_{0}$. In this picture, $P(N, \infty)=0(1)$ for a given $\left(p, M_{0}\right)$ implies that all such initial configurations ended in the absorbing state with only negative opinions (only positive opinions).

For $p>0.5$ [Fig. 6(a)], the dynamics reduces to a simple majority rule: the final state is completely ordered, following the opinion of the initial majority. The transition between the two absorbing states takes place at $\rho_{0}=0.5$. One should con-
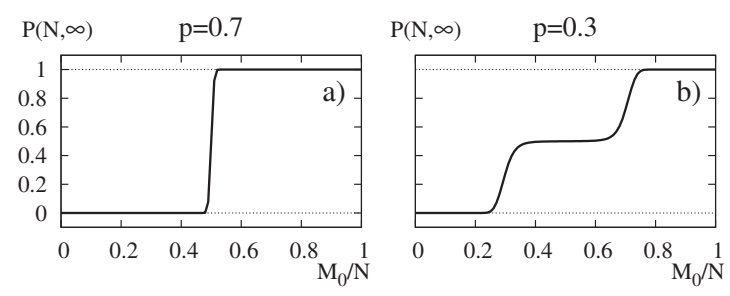

FIG. 6. The probability $P(N, \infty)$ to reach the state with $M=N$ positive spins at infinite times: $P(N, \infty)=0$ implies certain extinction of the positive population, while $P(N, \infty)=1$ represents a purely positive population. The figure displays two different lines which are not distinguishable due to the good agreement: (i) results obtained from direct iteration of the master equation [Eq. (2)] and (ii) the vector element $P(N, \infty)=\left(1-x_{M_{0}}\right) / 2$ from Eq. (30).

trast this with the "short time" behavior (Fig. 5), where a state corresponding to static opinions $\left[\rho(t)=\rho_{0}\right]$ appears if $\rho_{0} \in[1-p, p]$. On "long time" scales, we see that this state disappears. Eventually, the majority wins.

For $p<0.5$ [Fig. 6(b)], a small minority $\rho_{0}<p$ disappears, while a big majority $\rho_{0}>1-p$ wins the competition, as in the case of $N \rightarrow \infty$. A novel effect occurs for intermediate values of the initial positive population: $\rho_{0} \in[p, 1-p]$. Here, a fully disordered (but metastable) state develops on the short time scale, regardless of the initial condition. On long time scales, the system randomly falls into one of the two absorbing states. Thus, $P(N, \infty)=P(0, \infty)=0.5$ [Fig. 6(b)]. The initial population (of either opinion) is equally likely to become extinct. In this regime, consensus is achieved, but which opinion survives is completely random.

After having developed a qualitative picture, we analytically calculate the final state of the system with the exact probabilities $P(M, \infty)$ for arbitrary system size $N$. To do so, we write the master Eq. (2) in a matrix representation

$$
\partial_{t}|v(t)\rangle=\mathbb{L}|v(t)\rangle,
$$

where $|v(t)\rangle$ is the $N+1$ dimensional column vector with components $P(M, t), M=0,1, \ldots, N$, and the time evolution operator can be read off from Eq. (2) as

$$
\mathbb{L}=\left(\begin{array}{cccc}
0 & d_{1} & 0 & \ldots \\
b_{0} & -\left(b_{1}+d_{1}\right) & d_{2} & \\
0 & b_{1} & \ldots & \\
\ldots & & \ldots & d_{N} \\
& & b_{N-1} & 0
\end{array}\right) .
$$

Considering the symmetry in L, we have from Eqs. (7) and (8)

$$
b_{N-k}=d_{k} .
$$

The absorbing state condition is reflected in $b_{0}=d_{N}=0$.

The steady states of the system are the eigenvectors of $\mathbb{L}$. with zero eigenvalues. The two absorbing states correspond to the eigenvectors 


$$
\left|0_{0}\right\rangle \equiv\left(\begin{array}{c}
1 \\
0 \\
\vdots \\
0
\end{array}\right) \quad \text { and } \quad\left|0_{N}\right\rangle \equiv\left(\begin{array}{c}
0 \\
0 \\
\vdots \\
1
\end{array}\right)
$$

Each is already normalized in the sense that $\left\langle e \mid 0_{0, N}\right\rangle=1$, where $\langle e| \equiv(1,1, \ldots 1)$ is the vector used for normalization. To find their adjoints, it is convenient to study the symmetric and antisymmetric states

$$
\left|0_{ \pm}\right\rangle \equiv \frac{1}{2}\left\{\left|0_{0}\right\rangle \pm\left|0_{N}\right\rangle\right\}=\frac{1}{2}\left(\begin{array}{c}
1 \\
0 \\
\vdots \\
\pm 1
\end{array}\right),
$$

so that $\langle e|$, being symmetric, is naturally the adjoint of $\left|0_{+}\right\rangle$. For convenience, we can denote it as

$$
\left\langle 0_{+}\right| \equiv\langle e| .
$$

The adjoint of $\left|0_{-}\right\rangle$is an antisymmetric vector, given by $0=\left\langle 0_{-}\right| \mathbb{L}_{\text {L }}$. For $N=2 n$, there is an odd number of elements in this vector: $\left\langle 0_{-}\right| \equiv\left(\alpha_{0}, \alpha_{1}, \ldots \alpha_{n-1}, \alpha_{n},-\alpha_{n-1}, \ldots-\alpha_{1},-\alpha_{0}\right)$. For $N=2 n+1$, the adjoint contains $2 n+2$ elements, as follows: $\left\langle 0_{-}\right| \equiv\left(\alpha_{0}, \alpha_{1}, \ldots \alpha_{n},-\alpha_{n}, \ldots-\alpha_{1},-\alpha_{0}\right)$.

For $N=2 n$, the first $n$ equations $(k=0,1,2, \ldots, n-1)$, obtained from $0=\left\langle 0_{-}\right| \mathbb{L}$, read

$$
\begin{gathered}
0=\alpha_{0} d_{1}-\left(b_{1}+d_{1}\right) \alpha_{1}+b_{1} \alpha_{2} \\
\vdots \\
0=\alpha_{n-1} d_{n}-\left(b_{n}+d_{n}\right) \alpha_{n}-b_{n} \alpha_{n-1} .
\end{gathered}
$$

Due to the symmetry (20), the equations for $k=n, \ldots, 2 n$ are the same as for $k=0,1, \ldots, n-1$, and Eq. (24) is satisfied by $\alpha_{n}=0$ (since $b_{n}=d_{n}$ for this case). The remaining equations $(k=0, \ldots, n-2)$ can be written in the general form

$$
0=\alpha_{k-1} d_{k}-\left(b_{k}+d_{k}\right) \alpha_{k}+b_{k} \alpha_{k+1}
$$

or as a recursion

$$
d_{k} \Delta_{k-1}=b_{k} \Delta_{k} \quad \text { with } \quad \Delta_{k} \equiv \alpha_{k}-\alpha_{k+1} .
$$

Using the notation $r_{k} \equiv b_{k} / d_{k}$, we have

$$
\Delta_{k}=r_{k} \Delta_{k+1}=\prod_{\ell=k+1}^{n-1} r_{\ell} \Delta_{n-1}
$$

with $\Delta_{n-1}=\alpha_{n-1}$ which starts the recursion. Since

$$
\alpha_{j}=\sum_{k=j}^{n-1} \Delta_{k}=\sum_{k=j}^{n-1} \prod_{\ell=k+1}^{n-1} r_{\ell} \Delta_{n-1}
$$

we can express all the elements $\alpha_{j}$ in terms of a single unknown, $\alpha_{n-1}$,

$$
\begin{gathered}
\alpha_{n-2}=\left(r_{n-1}+1\right) \alpha_{n-1}, \\
\alpha_{n-3}=\left[\left(r_{n-2}+1\right) r_{n-1}+1\right] \alpha_{n-1}, \\
\vdots \\
\alpha_{0}=\left\{\left[\left(r_{1}+1\right) r_{2} \cdots+1\right] r_{n-1}+1\right\} \alpha_{n-1} .
\end{gathered}
$$

Since all the rates are positive, it is guaranteed that the $\alpha_{k}$ 's are monotonically decreasing in $k$. To construct the unique adjoint, we impose normalization $\left\langle 0_{-} \mid 0_{-}\right\rangle=1$ which means that

$$
\left\langle 0_{-}\right| \equiv\left(\begin{array}{lllll}
1 & x_{1} & x_{2} & \cdots & -x_{2}-x_{1}-1
\end{array}\right),
$$

where

$$
x_{j} \equiv \frac{\left\{\left[\left(r_{j+1}+1\right) r_{j+2}+1\right] r_{j+3} \cdots+1\right\} r_{n-1}+1}{\left\{\left[\left(r_{1}+1\right) r_{2}+1\right] r_{3} \cdots+1\right\} r_{n-1}+1}
$$

for $j=1, \ldots, n-1$ and $x_{n}=0$. Note that we automatically have $x_{j} \leq 1$.

For $N=2 n+1$, the central two equations read

$$
\begin{gathered}
0=\alpha_{n-1} d_{n}-\left(b_{n}+d_{n}\right) \alpha_{n}-b_{n} \alpha_{n}, \\
0=\alpha_{n} d_{n+1}+\left(b_{n+1}+d_{n+1}\right) \alpha_{n+1}-b_{n+1} \alpha_{n-1},
\end{gathered}
$$

which are the same when we take into account $d_{n+1}=b_{n}$ and $b_{n+1}=d_{n}$ due to the symmetry, Eq. (20). As for the start of the recursion, we now have $\Delta_{n}=2 \alpha_{n}$ instead of $\Delta_{n-1}=\alpha_{n-1}$, but the left eigenvector $\left\langle 0_{-}\right|$is still given by Eqs. (25) and (26). These will allow us to compute explicitly the final state

$$
\left|\psi_{\infty}\right\rangle \equiv \lim _{t \rightarrow \infty}\left|\psi_{t}\right\rangle
$$

Given an initial state $\left|\psi_{0}\right\rangle$, the solution to the master equation is

$$
\left|\psi_{t}\right\rangle=\sum_{\mu} e^{-\lambda} \mu^{t}|\mu\rangle\left\langle\mu \mid \psi_{0}\right\rangle,
$$

where $\langle\mu|$ and $|\mu\rangle$ are the left and right eigenvectors of $\mathbb{L}$ corresponding to the eigenvalues $\lambda_{\mu}$. Equation (28) projected into the space of zero eigenvalues gives

$$
\left|\psi_{\infty}\right\rangle=\left|0_{+}\right\rangle\left\langle 0_{+} \mid \psi_{0}\right\rangle+\left|0_{-}\right\rangle\left\langle 0_{-} \mid \psi_{0}\right\rangle .
$$

For example, if the initial state is a population with $M_{0}$ $<N / 2$ positive spins, the final state is

$$
\left|\psi_{\infty}\right\rangle=\left(\begin{array}{c}
P(0, \infty) \\
P(1, \infty) \\
\vdots \\
P(N-1, \infty) \\
P(N, \infty)
\end{array}\right)=\frac{1}{2}\left(\begin{array}{c}
1+x_{M_{0}} \\
0 \\
\vdots \\
0 \\
1-x_{M_{0}}
\end{array}\right) .
$$

This result shows that, indeed, in the $t \rightarrow \infty$ limit, the system arrives in one of its two absorbing states. Moreover, it provides the relative probabilities with which either will be reached, as a function of the initial $M_{0}$.

Finally, we plot the element $P(N, \infty)=0.5\left(1-x_{M_{0}}\right)$ of Eq. (30) for $N=100, p=0.7$ and $p=0.3$ in Figs. 6(a) and 6(b), 


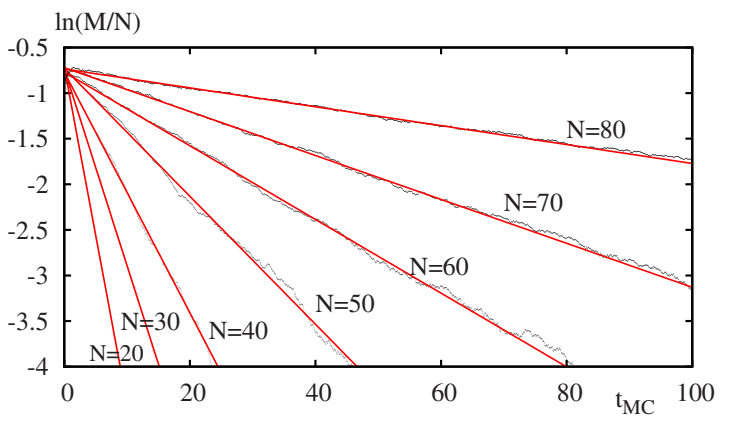

FIG. 7. (Color online) Relaxation of the disordered metastable state with $\rho=0.5$ to the completely ordered absorbing state $\rho=0$ for networks of different sizes. The simulation results (black lines) are averaged over 1000 runs. Straight (red) lines are the fitted exponentials. The parameters are $p=0.3, M_{0} / N=0.4$.

respectively. The agreement with the direct iteration of the master equation is so good that the two lines cannot be distinguished.

\section{LIFETIMES OF THE METASTABLE STATES}

In finite systems, the two metastable states ("disordered" and "static") will eventually relax to the absorbing states (full consensus). In this section, we investigate the time scales on which this relaxation occurs. Its dependence on the system size (number of nodes, $N$ ) will determine whether this relaxation can be observed on physical (or simulation) time scales or whether the metastable states will play the role of true thermodynamic phases, at least for all practical purposes.

The relaxation of the metastable state to the absorbing state is given by the smallest nonzero eigenvalue $\lambda$ of the time evolution operator $L_{\text {as }}$

$$
\rho \sim e^{-\lambda t}=e^{-t / \tau},
$$

where $\tau=1 / \lambda$ is the characteristic lifetime of the metastable state. Thus, we expect an exponential decay of the popularity $\rho$ to its value, $\rho_{\infty}$, as $t \rightarrow \infty$, or equivalently, an exponential decay of the positive population $M / N$. To check this relation, we performed numerical experiments for different systems ranging in size from $N=10$ to $N=80$. We choose as initial condition a value $\rho_{0} \in[p, 1-p]$ and $p<0.5$. In this case, the "disordered" metastable state with $\rho=0.5$ emerges first before the system relaxes to consensus. The results of the numerical simulations are presented in Fig. 7. Indeed, the relaxation is exponential and becomes slower with increasing system size. The decay rates $\lambda_{\text {sim }}(N)$ are easily obtained from the slopes of the straight lines in the log-normal plot.

We also evaluate the first nonzero eigenvalues, $\lambda(N)$, of the time evolution operator $\mathbb{L}$, through direct (numerical) diagonalization of Eq. (2). These eigenvalues, evaluated for systems of size $N=10,20, \ldots, 80$, are plotted in Fig. 8 along with the simulated rates, $\lambda_{\text {sim }}(N)$, showing a good agreement. As the system size increases, the decay rates decrease exponentially, indicating that the lifetime $\tau$ increases with $N$ as

$$
\tau \sim e^{a\left(p, M_{0}\right) N} .
$$
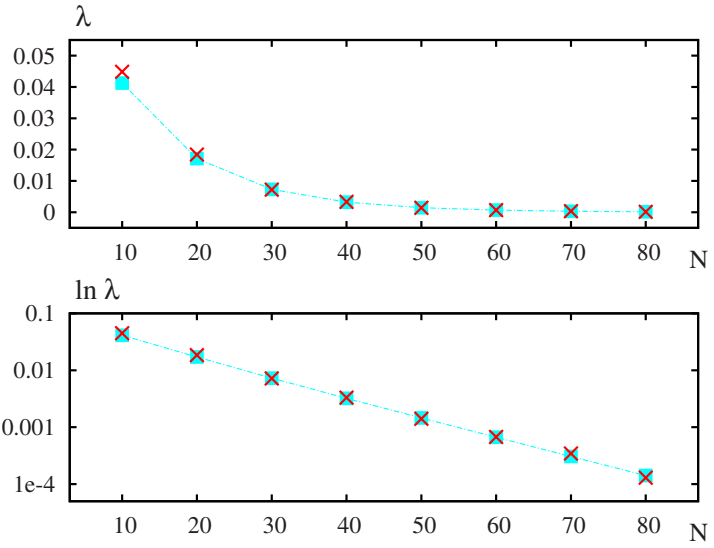

FIG. 8. (Color online) The first nonzero eigenvalues (blue squares) of the time evolution operator $\mathrm{L}$ and the slopes of the relaxations plotted in Fig. 7 (red crosses), as a function of the system size. The parameters are the same as in Fig. 7.

This behavior holds for the relaxation from both the disordered and the static metastable states into the absorbing states, as illustrated in Figs. 9(a) and 9(b). The prefactor $a(p)$ in the exponential depends on the value of the parameter $p$. For $p=0.3$ and $M_{0} / N=0.4$, we find $a\left(p, M_{0}\right) \simeq 0.08$, and for $p=0.8$ and $M_{0} / N=0.4$, the result is $a\left(p, M_{0}\right) \simeq 0.11$. In conclusion, we note that all times here are measured relative to Monte Carlo time, $t_{\mathrm{MC}}$.

A simple estimate of the time to consensus for a network of $N=1000$ voters with $p=0.3$ shows that it may take as many as $10^{36}$ spin flips to reach one of the absorbing states. Since human societies are much larger, consensus is practically impossible and the metastable states will persist for practically infinite times.

\section{GENERALIZATION TO $q \neq 1-p$}

In the previous sections, we discussed in some detail a case with a special symmetry: links between agents holding similar opinions are present (absent) with probability $p(1-p)$ and links between agents holding opposite opinions are present (absent) with probability $q \equiv 1-p(1-q \equiv p)$. Of course, this symmetry does not naturally appear in real social systems. There is no a priori reason to assume any kind of relation between the probabilities $p$ and $q$, and in general, they should be considered as independent parameters of the model.
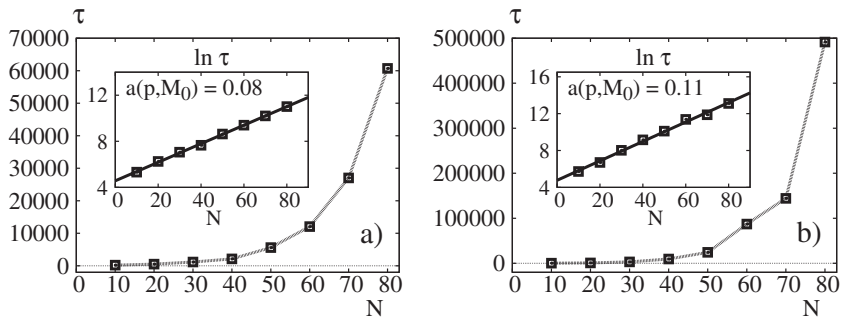

FIG. 9. Time $\tau$ needed to reach consensus vs system size $N$ for (a) $p=0.3, M_{0} / N=0.4$ and (b) $p=0.8, M_{0} / N=0.4$. Insets show the exponential dependence on $N$. 

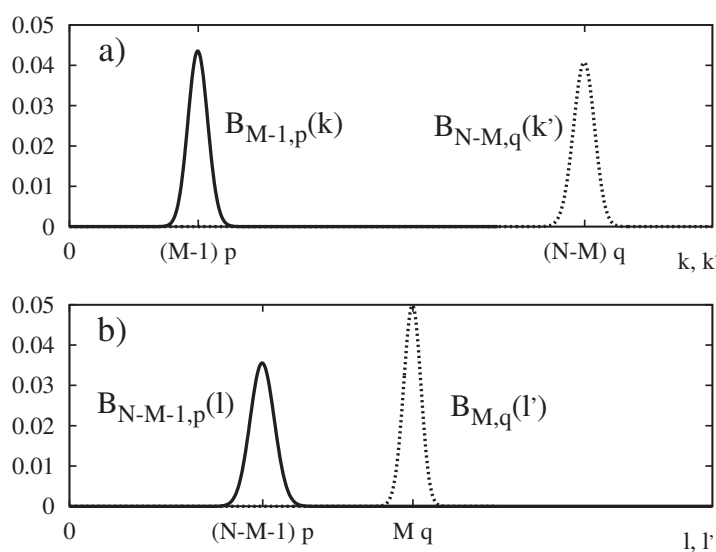

FIG. 10. Illustration of the binomial distributions appearing in (a) Eq. (4) and (b) Eq. (6). Parameters used for the plot are $p$ $=0.3, q=0.5, M=400$, and $N=1000$.

Given that we have an intuitive and quantitative picture of the opinion dynamics for $q=1-p$, we can easily generalize our analysis for arbitrary $q \neq 1-p$. In the general case, the transition rates in the master equation, Eq. (2), are given by Eqs. (4) and (6). The simplification, to Eqs. (7) and (8), is no longer possible, but we can still obtain intuitive insight into these rates. Also, needless to say, they are easily computed numerically. First, we consider the death rate $d_{M}$, for large systems $(N \rightarrow \infty)$, and evaluate the sums approximately. If the centers of the two binomials, $(M-1) p$ and $(N-M) q$, are further apart than the sum of their half widths, given by $1 / 2 \sqrt{(M-1) p(1-p)}+1 / 2 \sqrt{(N-M) q(1-q)}$, the typical situation is depicted in Fig. 10(a).

To continue, we recall that $d_{M}$ is associated with picking a positive spin and counting the number of connections to positive $(k)$ and negative $\left(k^{\prime}\right)$ spins. Since the two binomials are far apart, they select the values of $k$ and $k^{\prime}$ from two well-separated regions. Hence, we can easily decide whether $k<k^{\prime}$ or not, i.e., whether the spin will flip or not. Thus, we can eliminate the $\Theta$ function from Eq. (4) and obtain

$$
d_{M} \simeq \frac{M}{N} \begin{cases}1 & \text { if } M p<(N-M) q \\ 0 & \text { if } M p>(N-M) q .\end{cases}
$$

In a similar fashion we can eliminate the $\Theta$ function from the expression Eq. (6) for the birth rate $b_{M}$ [see Fig. 10(b)], giving

$$
b_{M} \simeq \frac{N-M}{N} \begin{cases}0 & \text { if }(N-M) p<M q \\ 1 & \text { if }(N-M) p>M q .\end{cases}
$$

Thus, the sums in Eqs. (4) and (6) will behave as the Gaussian error function and its complementary error function (see Fig. 11), similar to the special case discussed previously. The dependences of the transition rates on $M / N$, for a few selected values of $p$ and $q$, are shown in Fig. 12.

We can see immediately that the late-time properties of the model, the relaxations to the steady states, the existence of the metastable states, and the particular forms and solutions of the master equation in the different regimes are com-

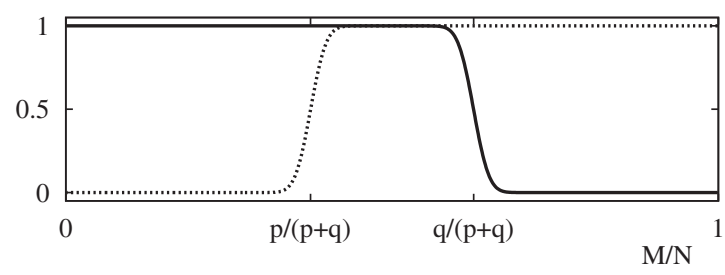

FIG. 11. The cumulative distribution functions appearing in Eqs. (6) and (4) (solid and dotted lines, respectively).

pletely analogous to the special case $q=1-p$. The only difference is a shift in the phase boundaries. Their locations can be obtained from Eqs. (33) and (34) as

$$
\rho_{0}=\frac{p}{p+q} \quad \text { and } \quad \rho_{0}=\frac{q}{p+q} .
$$

For probabilities $p<q$ and $\rho_{0}<p /(p+q)$, the pure death process as in Eq. (12) leads to the extinction of the positive population and the state $\rho_{\infty}=0$ is reached exponentially as described by Eq. (13). If $\rho_{0}>q /(p+q)$, we have a pure birth process and the system relaxes exponentially to the state $\rho_{\infty}=1$. In the intermediate region $\rho_{0} \in[p /(p+q), q /(p+q)]$, the system reaches exponentially the disordered phase $\rho_{\infty}$ $=0.5$, with a characteristic time scale $1 / 2$.

For probabilities $p>q$, a small minority $\rho_{0}<q(p+q)$ will become extinct and a large majority $\rho_{0}>q /(p+q)$ will win. In the interval $\rho_{0} \in[q /(p+q), p /(p+q)]$, the fraction of positive spins remains frozen at its initial value.

The phase diagram in the $\left(p, \rho_{0}\right)$ plane, along with the phase boundaries, is shown in Fig. 13 for different choices of the parameter $q$. Larger values of $q$ indicate a greater willingness to connect with individuals holding an opinion opposed to ones own. This behavior extends the region in which the opinions balance ( $\rho=1 / 2$, the "disordered" phase). For smaller $q$, most interactions tend to be restricted to people with similar beliefs and so the society tends to become more "static."

With regards to finite-size effects, our study in Sec. V did not rely on any assumptions regarding the specific form of the birth and death rates. Hence, the analytic results obtained there remain unchanged. In finite systems, the metastable states will still decay on long time scales and consensus will be reached. The relative probabilities of the absorbing states are still given by the same expression, Eq. (30).

\section{CONCLUSIONS}

In this study, we proposed and investigated an adaptive model for opinion dynamics in which the agents and the

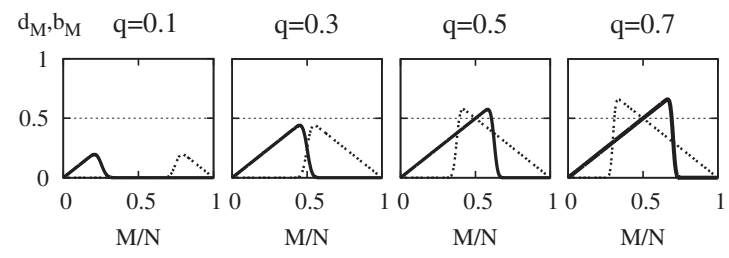

FIG. 12. The death $d_{M}$ and birth $b_{M}$ rates (solid and dashed lines, respectively) given by Eqs. (33) and (34) for different probabilities $q$ and $p=0.3$. For fixed $p$ and $q$, there are three different regimes of behavior bounded by Eq. (35). 

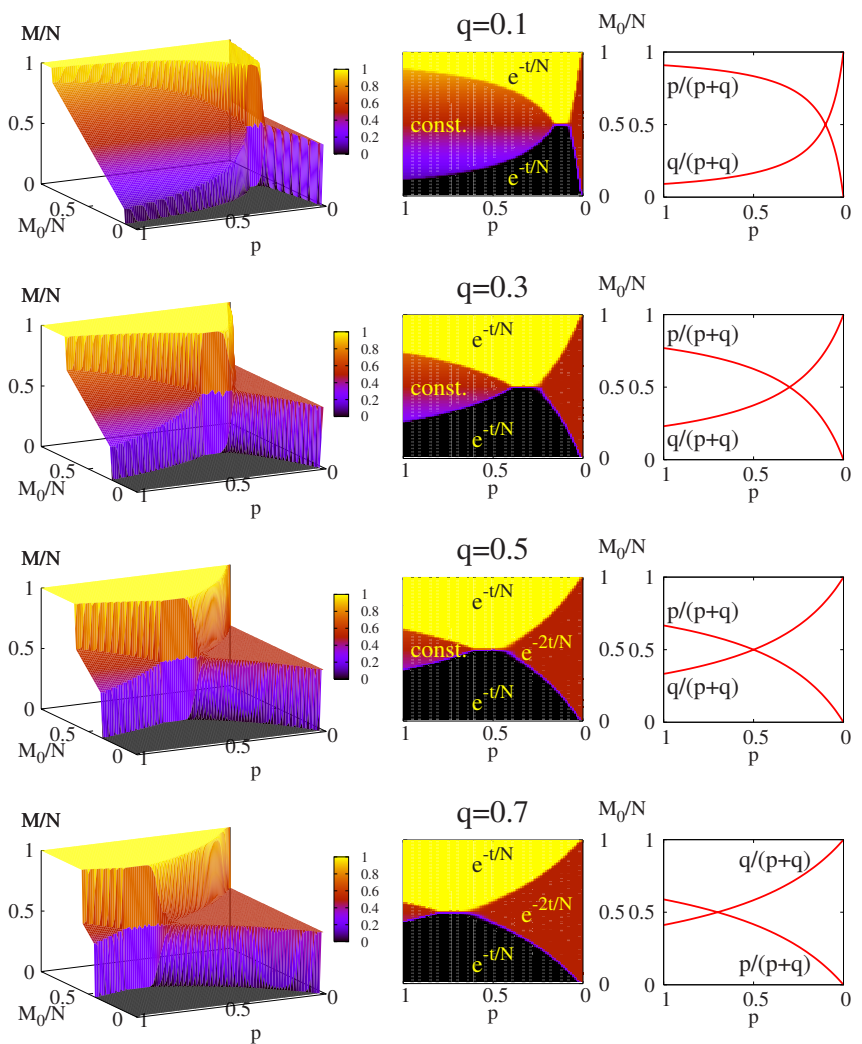

FIG. 13. (Color online) The fraction of positive spins $M / N$ after $t_{\mathrm{MC}}=10$, as function of $p$ and $M_{0} / N$ for a network of $N=1000$ nodes in a three-dimensional plot (left panels) and a twodimensional projection (center panels), for different probabilities $q$. The right panels show the phase boundaries given by Eq. (35).

underlying network structure coevolve. The opinions of the agents determine their connections, and these connections, in turn, play an important role for how an agent changes his or her opinion.

Our conclusions are most easily understood in terms of a two-party system. In large societies, the best strategy for a minority group is to establish many contacts with its opponents. In this way, it can convince half of them and keep this balance for a long time. If the same minority group is less open for discussions, it cannot overcome the majority, but at least it will not disappear. It is tempting to speculate how these results might be applied to real social systems. Will two-party systems, once formed, persist for long times? Will bilingual regions remain bilingual? Will relatively isolated parties continue to receive the same, almost constant percentage of the vote? Will closed religious communities continue to exist without gaining or losing members?

Our findings depend drastically on the size of the system. In large systems, coexistence of different opinions is typical and persists for long times. In contrast, in smaller systems, consensus is reached after much shorter times. This may be an explanation for an empirical study of the number of languages in the Solomon Islands [34]. It was found that small islands (less than 100 square miles) have a single language, but on islands of larger size, the number of languages increases.

Even though our model neglects certain social factors (e.g., spatial and age structures, a spectrum of opinions, etc.) and it is defined for one particular type of agent dynamics, its attraction rests in its mathematical simplicity which allows an analytical investigation of the properties. Our model proposes a mechanism for characterizing the adaptive nature of interpersonal relations in social models and can thus serve as a "baseline model" for such systems. Of course, many questions remain open and should be investigated in order to place our findings into a broader context. For example, in the dynamics considered here, the topology of the network has no memory: after each spin flip, all links are removed and re-established according to the probabilities $p$ and $q$. If links are left unchanged unless one of the two associated nodes are updated or if links are updated only after several spin updates have occurred, the dynamics acquires a (short-term) memory. Preliminary results indicate that the phase diagram remains unchanged but the relaxation into the different phases is affected. Another interesting issue concerns the presence of noise, especially if it allows individuals to change their opinions spontaneously, with a small probability (corresponding to a finite temperature in the spin picture). This would certainly eliminate the absorbing states, replacing them with stationary states characterized by a small but finite density of the minority opinion. It is not clear how the presence of this type of noise would affect the metastable states. Certainly, it might increase the possibility of a large fluctuation which would drive the system toward the stable state. In future work, we will also combine the adaptive nature of the network presented here with other types of agent dynamics (for instance epidemic dynamics or Axelrod opinion dynamics) and the type of underlying network as well as the dynamics of the connections can also be refined. For example, the network can be given a spatial structure (a "metric") or the links can be directed or heterogeneous, reflecting the importance of different personality types. Clearly, many open questions remain for further study.

\section{ACKNOWLEDGMENTS}

We thank S. Redner, M. Mobilia, and I. Georgiev for useful discussions and K. Holsinger for the binomial generator. This research is supported in part by the National Science Foundation through Grant No. DMR-0705152 and the College of Science at Virginia Tech.

\section{APPENDIX: THE BIRTH AND DEATH RATES}

Here, we compute the double sum appearing in Eq. (4). It involves the product of two binomial distributions. Labeling the two (independent) random variables $X_{1}$ and $X_{2}$, taking values $k=0,1, \ldots, M-1$ and $k^{\prime}=0,1, \ldots, N-M$, respectively, the two corresponding probability distributions are given by

$$
B_{M-1, p}(k)=\frac{(M-1) !}{k !(M-1-k) !} p^{k}(1-p)^{M-1-k} .
$$




$$
B_{N-M, 1-p}\left(k^{\prime}\right)=\frac{(N-M) !}{k^{\prime} !\left(N-M-k^{\prime}\right) !} p^{N-M-k^{\prime}}(1-p)^{k^{\prime}} .
$$

According to Eq. (4), the selected spin will flip if $k^{\prime}-k>0$. This suggests that we consider the distribution of the difference, $D \equiv X_{2}-X_{1}$, defined via

$$
P_{D}(K) \equiv \sum_{k=0}^{M-1} \sum_{k^{\prime}=0}^{N-M} B_{M-1, p}(k) B_{N-M, 1-p}\left(k^{\prime}\right) \delta_{K, k^{\prime}-k} \text {. }
$$

We now invoke a well-known result from the theory of generating functions. Defining $G_{D}(z)$, the generating function associated with $P_{D}(K)$, via

$$
G_{D}(z) \equiv \sum_{K} P_{D}(K) z^{K}
$$

and $g_{X_{1}}(z)$ and $g_{X_{2}}(z)$, associated with $B_{M-1, p}(k)$ and $B_{N-M, 1-p}\left(k^{\prime}\right)$, via

$$
g_{X_{1}}(z) \equiv \sum_{k} B_{M-1, p}(k) z^{k}=[(1-p)+p z]^{M-1},
$$

$$
g_{X_{2}}(z) \equiv \sum_{k^{\prime}} B_{N-M, 1-p}\left(k^{\prime}\right)=[(1-p) z+p]^{N-M},
$$

we can write the generating function of the difference variable in terms of $g_{X_{1}}(z)$ and $g_{X_{2}}(z)$,

$$
G_{D}(z)=g_{X_{1}}(z) g_{X_{2}}(1 / z) .
$$

Inserting the explicit expressions, we obtain

$$
G_{D}(z)=[(1-p)+p z]^{N-1}(1 / z)^{N-M},
$$

where the first factor is easily recognized as the generating function of a binomial distribution, $B_{N-1, p}(\ell)$, and the second factor is associated with a constant distribution, $p(s)$ $=\delta_{s, N-M}$. The variable $\ell$ can take values $\ell=0,1, \ldots, N-1$, while the difference variable $D$ is shifted to the left from $\ell$ by $N-M$, taking values $K=\ell-(N-M)=-N+M,-N+M$ $+1, \ldots, M-1$ with probability $B_{N-1, p}(\ell)$. Since expression (4) contains only terms for which $K<0$, we have to sum up only the first $N-M$ terms, i.e.,

$$
d_{M}=\frac{M}{N} \sum_{K=0}^{N-M-1} B_{N-1, p}(K) .
$$

Following similar considerations, Eq. (6) results in Eq. (8).
[1] C. Castellano, S. Fortunato, and V. Loreto, arXiv:0710.3256.

[2] T. M. Liggett, Stochastic Interacting Systems: Contact, Voter, and Exclusion Processes (Springer, New York, 1999).

[3] P. L. Krapivsky, Phys. Rev. A 45, 1067 (1992).

[4] L. Frachebourg and P. L. Krapivsky, Phys. Rev. E 53, R3009 (1996).

[5] E. Ben-Naim, L. Frachebourg, and P. L. Krapivsky, Phys. Rev. E 53, 3078 (1996).

[6] I. Dornic, H. Chaté, J. Chave, and H. Hinrichsen, Phys. Rev. Lett. 87, 045701 (2001).

[7] R. Durrett and S. A. Levin, Philos. Trans. R. Soc. London, Ser. B 343, 329 (1994).

[8] M. Nowak and R. May, Nature (London) 359, 826 (1992); Int. J. Bifurcation Chaos Appl. Sci. Eng. 3, 35 (1993); R. Durrett, SIAM Rev. 41, 677 (1999).

[9] P. L. Krapivsky and S. Redner, Phys. Rev. Lett. 90, 238701 (2003).

[10] M. Mobilia and S. Redner, Phys. Rev. E 68, 046106 (2003).

[11] S. Galam, Physica A 333, 453 (2004).

[12] K. Sznajd-Weron and J. Sznajd, Int. J. Mod. Phys. C 11, 1157 (2000).

[13] C. Castellano, M. Marsili, and A. Vespignani, Phys. Rev. Lett. 85, 3536 (2000).

[14] M. Mobilia, Phys. Rev. Lett. 91, 028701 (2003).

[15] F. Vazquez, P. L. Krapivsky, and S. Redner, J. Phys. A 36, L61 (2003).

[16] K. Klemm, V. M. Eguiluz, R. Toral, and M. San Miguel, Phys. Rev. E 67, 045101(R) (2003).

[17] C. J. Tessone, R. Toral, P. Amengual, H. S. Wio, and M. San
Miguel, Eur. Phys. J. B 39, 535 (2004).

[18] R. Dickman and R. Vidigal, J. Phys. A 35, 1147 (2002).

[19] F. Slanina and H. Lavicka, Eur. Phys. J. B 35, 279 (2003).

[20] S. Galam, Eur. Phys. J. B 25, 403 (2002).

[21] K. Suchecki, V. M. Eguiluz, and M. San Miguel, Europhys. Lett. 69, 228 (2005).

[22] C. Castellano, D. Vilone, and A. Vespignani, Europhys. Lett. 63, 153 (2003).

[23] D. Vilone and C. Castellano, Phys. Rev. E 69, 016109 (2004).

[24] F. Vazquez, V. M. Eguíluz, and M. S. Miguel, Phys. Rev. Lett. 100, 108702 (2008).

[25] C. Nardini, B. Kozma, and A. Barrat, Phys. Rev. Lett. 100, 158701 (2008).

[26] Feng Fu and Long Wang, Phys. Rev. E 78, 016104 (2008).

[27] S. Gil and D. Zanette, Phys. Lett. A 356, 89 (2006).

[28] F. Vazquez and S. Redner, Europhys. Lett. 78, 18002 (2007).

[29] M. Liu and K. E. Bassler, Phys. Rev. E 74, 041910 (2006).

[30] A. E. Allahverdyan and K. G. Petrosyan, Europhys. Lett. 75, 908 (2006).

[31] P. Holme and M. E. J. Newman, Phys. Rev. E 74, 056108 (2006).

[32] I. J. Benczik, S. Z. Benczik, B. Schmittmann, and R. K. P. Zia, Europhys. Lett. 82, 48006 (2008). For the convenience of the reader, some of the figures from that article are reproduced here.

[33] The binomial generator used the BTPEC algorithm described in V. Kachitvichyanukul and B. W. Schmeiser, Commun. ACM 31, 216 (1988).

[34] J. Terrel, Fieldiana Anthropology 8, 1 (1977). 